\title{
Endogenous temporal orienting of attention in detection and discrimination tasks
}

\author{
ÁNGEL CORREA and JUAN LUPIÁÑEZ \\ Universidad de Granada, Granada, Spain \\ BRUCE MILLIKEN \\ McMaster University, Hamilton, Ontario, Canada \\ and \\ PÍO TUDELA \\ Universidad de Granada, Granada, Spain
}

\begin{abstract}
Endogenous temporal-orienting effects were studied using a cuing paradigm in which the cue indicated the time interval during which the target was most likely to appear. Temporal-orienting effects were defined by lower reaction times (RTs) when there was a match between the temporal expectancy for a target (early or late) and the time interval during which the target actually appeared than when they mismatched. Temporal-orienting effects were found for both early and late expectancies with a detection task in Experiment 1. However, catch trials were decisive in whether temporal-orienting effects were observed in the early-expectancy condition. No temporal-orienting effects were found in the discrimination task. In Experiments $2 \mathrm{~A}$ and 2B, temporal-orienting effects were observed in the discrimination task; however, they were larger when temporal expectancy was manipulated between blocks, rather than within blocks.
\end{abstract}

Focusing attention toward the location of an object in a visual scene is important for adaptive behavior in many contexts. Some authors have proposed that processing the spatial location of stimuli has a special status for our cognitive system (e.g., Garner, 1987). Posner's costs and benefits paradigm has been used by many researchers to study the orienting of visual attention in space (see also Posner, Nissen, \& Ogden, 1978; Posner, Snyder, \& Davidson, 1980). Studies in which this paradigm has been used have shown that previously directing attention to a location benefits performance for a target that appears in that same location in a variety of tasks. This result suggests that processing of the target is modulated in some way by the orienting of attention to that location. This attentional modulation can be observed not only behaviorally, in faster and/or more accurate responses for attended targets, but also physiologically, in increased cerebral blood flow and altered electrical potentials in relevant areas of

This research was financially supported by the Spanish Ministerio de Educación y Cultura with Predoctoral Grant FPU-AP2000-3167 to the first author and Research Grants MCyT, BSO2000-1503 to J.L. and MCyT, BSO2000-1411-C02to P.T. We thank Albrecht Inhoff, Sander A. Los, and an anonymous reviewer for their helpful comments on the manuscript. Correspondence concerning this article should be addressed to Á. Correa or J. Lupiáñez, Departamento de Psicología Experimental y Fisiología del Comportamiento, Facultad de Psicología, Universidad de Granada, Campus Universitario de Cartuja s/n, 18071 Granada, Spain (e-mail: act@ugr.es or jlupiane@ugr.es).

Note-This article was accepted by the previous editorial team, headed by Neil Macmillan. the visual system (e.g., Posner \& Raichle, 1994). These findings are traditionally interpreted in terms of the spotlight metaphor. According to this view, stimuli that appear within an attentional spotlight are allocated more resources than are stimuli that appear outside that attentional spotlight.

Analogous to the orienting of attention in space, researchers have begun to consider the possibility that attention also can be focused to a specific moment in time (Coull \& Nobre, 1998; Kingstone, 1992). The temporal dimension is clearly relevant in perception. Kubovy (1981) has argued that space and time are both "indispensable attributes" of perception. It seems likely that we take into account temporal information to fix ourselves in a particular context within our dynamic and complex environment. From this perspective, temporal expectancy about when an event will occur is also likely to benefit our behavior.

According to Coull and Nobre (1998), attentional orienting in time is the ability to use information about time intervals "to direct attention to a point in time when a relevant event is expected, to optimize behavior" (p. 7426). Behaviorally, a temporal-orienting effect can be defined as a decrease in reaction time (RT) as a consequence of a match between temporal expectancy for an event and the actual temporal occurrence of that event. In other words, RTs that are shorter when the target appears at expected than when it appears at unexpected time intervals can be attributed to temporal-orienting processes. This definition is consistent with effects that have been found 
previously in the field of alertness. When a warning signal was presented in simple-RT (Woodrow, 1914) or choice-RT (Bertelson, 1967) tasks, an improvement in RTs was found. This RT improvement was interpreted as being caused by participants' certainty about the moment when the target would appear, which they gained by using the warning signal as a temporal cue. Presumably, the participants used the warning signal to make "preparatory adjustments" in advance of the occurrence of the stimulus (Bertelson, 1967).

However, Coull and Nobre (1998) claimed that the alertness effect in the experiments with warning signals was not produced by a rigid mechanism. Rather, they proposed that attentional resources can be allocated dynamically to a particular time interval. In this sense, the mechanism underlying temporal orienting of attention can be endogenous - that is, voluntarily controlled by the cognitive system. Thus, a warning signal could trigger two different processes at the same time: an alertness process and an attentional preparation process. The former involves a general increase of activation in organisms (i.e., an unspecific preparation; Posner, 1978). The latter involves, according to LaBerge (1995), "an elevation of activity in the corresponding perceptual or action brain area that speeds processing of stimuli or actions when the appropriate triggering event occurs" (p. 51) that is, a specific preparation based on a temporal expectancy. Recently, other authors have proposed another factor that may contribute to unspecific preparation: The warning signal could act as a conditioned stimulus that triggers a preparatory response, which is time locked to the unconditioned stimulus (i.e., the target) and is learned by trace conditioning (Los \& Van den Heuvel, 2001).

In this sense, some authors have differentiated between exogenous and endogenous processes in temporal orienting (Coull, Frith, Büchel, \& Nobre, 2000; Milliken \& Lupiáñez, 2000). Exogenous processes are related to a rigid mechanism of alertness (or trace conditioning), whereas endogenous processes are related to an attentional preparation process that can be modulated by strategic factors, such as temporal expectancies.

In several studies of preparation in which the foreperiod (FP) has been manipulated (see Niemi \& Näätänen, 1981, for a review), one can find additional evidence supporting the idea that participants spontaneously use temporal expectancies to guess the moment at which a target will appear (Alegria, 1975). However, Coull and Nobre (1998) analyzed directly how a temporal expectancy can modulate the process of preparation. They presented an endogenous cue that provided predictive temporal information, so that participants could anticipate whether the stimulus was likely to appear after a short $(300 \mathrm{msec})$ or a long $(1,500 \mathrm{msec})$ interval. Then the target appeared either in the cued interval with a probability of .80 (valid trials) or in the uncued interval with a probability of .20 (invalid trials). The results were analogous to those from spatial orienting studies: Stimuli that appeared in the cued time interval were detected more quickly than those that appeared in the uncued in- terval. This effect was attributed to temporal orienting, since participants were presumed to have used the temporal information provided by the cue to predict the likely temporal onset of the target. They were encouraged to adopt this orienting strategy by the high predictability of the cue (.80). Presumably, the participants decoded the symbolic meaning of the cue and used it to generate a temporal expectancy, directing attention voluntarily to the cued interval. This orienting process had to be flexible and dynamic, since the time interval changed from trial to trial.

A review of the research literature on endogenous temporal orienting (Coull et al., 2000; Coull \& Nobre, 1998; Coull, Nobre, \& Frith, 2001; Milliken, Lupiáñez, Roberts, \& Stevanovski, 2003; Miniussi, Wilding, Coull, \& Nobre, 1999) reveals that orienting effects for both early and late expectancies are rarely found (Milliken et al., 2003; Miniussi et al., 1999). Instead, orienting effects are often reported only for the late expectancy. Yet observing a temporal-orienting effect for both early and late expectancies is important for the argument that the underlying mechanism is endogenous in nature, since orienting effects for just a late expectancy could be explained by alertness processes.

Several authors (e.g., Alegria, 1975; Coull \& Nobre, 1998; Karlin, 1959; Kingstone, 1992; Loveless \& Sanford, 1974; McAuley \& Kidd, 1998) have discussed a reorienting or repreparing process, which could explain why temporal-orienting effects are often not observed for an early expectancy (but see Los \& Van den Heuvel, 2001 , for an account different from the reorienting one). It is assumed that reorienting can occur when participants have an early expectancy and the target fails to appear early. In response to this failed prediction, participants may reorient their attention to the long interval. Consequently, despite the cue's indicating a highly probable early target, participants may be as well prepared for a target at the long interval as for a target at the short interval. This conclusion is supported by the fact that RTs are often not shorter on early-expectancy-short-interval trials than on early-expectancy-long-interval trials.

The reorienting hypothesis is well supported by physiological (Loveless \& Sanford, 1974) and neuroimaging (Coull et al., 2000) studies. However, the reorienting process has never been directly manipulated to study its influence on temporal orienting. In the present study, we examined whether it is possible to gain empirical control over this reorienting process. How might this empirical control be achieved?

A straightforward answer to this question is to gain control over whether participants act on the knowledge that if the target doesn't appear at the short interval, it necessarily will appear at the long interval. Methodologically, we can eliminate this contingency by including trials in which the target does not appear (i.e., catch trials). Indeed, in experiments in which temporal-orienting effects have been reported for an early expectancy, catch trials have been part of the design (Milliken et al., 2003; Miniussi et al., 1999). According to our hypothesis, the 
presence of catch trials implies that the target cannot be confidently expected at the long interval when it does not appear at the short interval. This uncertainty may well be sufficient to prevent participants from confident predictions about the appearance of the target, which we presume to be the basis of the reorienting process. Thus, when an early expectancy is violated in the context of an experiment with catch trials, RTs may be long when the target appears at a long interval, because the reorienting process is not engaged.

Thus, the primary aim of the present study was to examine whether the presence/absence of catch trials dictates whether reorienting occurs in the context of a temporalorienting study.

One difference between our procedure and that of Coull and Nobre (1998) was the inclusion of a third stimulus onset asynchrony (SOA). This additional SOA was used to examine whether the orienting of attention produces effects on RT that depend on the difference between the cued (expected) temporal interval and the actual temporal interval. Since this property holds for the orienting of spatial attention (Shulman, Sheehy, \& Wilson, 1986), and given that this property has been observed in one other temporal-orienting study (Milliken et al., 2003), we expected a progressive increase in RTs as the difference between expected and actual SOAs became larger.

Finally, we examined whether temporal orienting occurs in discrimination tasks, since it has been fundamentally studied with simple-RT tasks (but see Griff in, Miniussi, \& Nobre, 2001, Kingstone, 1992, or Los \& Van den Heuvel, 2001). Thus, if temporal-orienting effects are due to the orienting of attention in time, effects should be general and ought to be found across different tasks. In contrast, if the effects are due to a specific motor preparation, they should be more easily observed in those tasks in which a simple and direct stimulus-response ( $\mathrm{S}-\mathrm{R})$ mapping is used, as in simple detection tasks. Thereby, it could be interesting, in parallel with studies of spatial attention, to explore whether effects of attentional orienting generalize to choice-RT tasks, such as our shape discrimination task.

Moreover, the use of shape discrimination tasks could be useful for future research with physiological measures to test more adequately the possibility that temporal orienting might modulate early visual processing, since such tasks demand a more detailed perceptual analysis than do detection tasks. However, at the moment, there is no neuroimaging research with other tasks different from detection tasks. Therefore, the studies that suggest that temporal orienting exclusively affects late stages of processing, such as preparation of a motor response (Coull et al., 2000; Coull \& Nobre, 1998; Miniussi et al., 1999), would be better contrasted with the use of shape discrimination tasks.

\section{EXPERIMENT 1}

In this experiment, the influence of uncertainty on endogenous temporal orienting was explored by manipu- lating the presence/absence of catch trials. In addition, temporal orienting was studied in both detection and shape discrimination tasks.

According to the definition of temporal orienting proposed above, temporal-orienting processes ought to produce an interaction between temporal expectancy and SOA. Furthermore, we expected to observe a significant interaction between temporal expectancy and SOA in both detection and discrimination tasks. Concerning the effect of catch trials, we expected that it would modulate the influence of the temporal cue. In particular, without catch trials, we predicted that an expect-early cue would not necessarily produce faster responses for early than for late targets. This prediction was based on the notion that, when there are no catch trials, participants may reorient to a late temporal interval when the target does not appear at the cued early temporal interval.

In contrast, with catch trials, participants ought to produce faster responses for early targets when given an expect-early cue. This prediction follows because participants will be less likely to reorient to a long interval when such reorientation is often met with the absence of a target.

\section{Method}

\section{Participants}

Thirty-two students of psychology took part in the experiment for course credit. There were four groups composed of 8 participants each.

\section{Apparatus and Stimuli}

MEL software (Schneider, 1988) controlled the presentation of stimuli and data collection. The experiment was run on a 486/33 processor connected to a 14-in. VGA monitor. All the stimuli appeared in the center of the screen. The stimuli that appeared on each trial included a fixation point ("+" symbol), an endogenous cue, and a target. The endogenous cue was either a red or a green square, $14 \mathrm{~mm}$ in width $\times 17 \mathrm{~mm}$ in height, subtending visual angles of $1.34^{\circ}$ and $1.62^{\circ}$, respectively, at a viewing distance of $60 \mathrm{~cm}$. The target was either the letter "O" or the letter "X," $4 \mathrm{~mm}$ in width $\times$ $8 \mathrm{~mm}$ in height, subtending visual angles of $0.38^{\circ}$ and $0.76^{\circ}$, respectively. The two target letters appeared with a probability of .50 . In the detection task group, the participants pressed the "B" key when either an "O" or an "X" appeared. In the discrimination task group, the participants pressed the " $Z$ " key for one target and the "M" key for the other target. The assignment of targets to response keys was counterbalanced across participants within each group.

\section{Procedure}

The participants sat approximately $60 \mathrm{~cm}$ from the screen. Instructions concerning the task were displayed on the screen at the beginning of the experimental session. The participants were explicitly informed that the temporal cue would help them to predict when the target would appear. Half of the participants were told that the green square indicated that the target would appear early, whereas the red square indicated that the target would appear late. The meanings associated to the colors of the cues were counterbalanced. So, for the remaining half of the participants, the red square indicated that the targets were likely to appear early, whereas the green square indicated that targets were likely to appear late. Auditory feedback (a 400-Hz tone of $100 \mathrm{msec}$ ) was provided on error trials. The participants were encouraged to respond as quickly and accurately as possible. The group with catch trials was not informed about the presence of catch trials. 
A sketch of the sequence of events on a trial is depicted in Figure 1.

The fixation point was displayed in black on a gray background for a random interval between 500 and 1,500 msec. The endogenous cue, a red or a green square, then appeared for $50 \mathrm{msec}$. The cue was then removed, and the screen remained blank for a variable delay of 350,850 , or $1,350 \mathrm{msec}$, depending on the SOA for that trial. Finally, the target was displayed for $100 \mathrm{msec}$ and was then replaced by a blank screen until the detection or discrimination response. After the response, the next trial began. When no response was made, the next trial began after a delay of $2,000 \mathrm{msec}$.

The experimental session lasted about $30 \mathrm{~min}$. Each session included one block of 64 practice trials and four blocks of 128 experimental trials. There was a 1-min interruption for rest at the end of each block.

In the group with uncertainty (i.e., with catch trials), there were 32 trials without a target and 96 trials with a target, thus producing a catch trial proportion of .25 . The 96 target trials consisted of 72 validly cued trials and 24 invalidly cued trials, thus producing a validity proportion of .75 . On half of the valid trials, the cue indicated that the target was likely to appear early, and the target appeared $400 \mathrm{msec}$ after cue onset. On the other half of the valid trials, the cue indicated that the target was likely to appear late, and the target appeared $1,400 \mathrm{msec}$ after cue onset. The invalid trials were equally distributed between the two uncued SOAs for both early and late cues. Thus, all the trials at the medium SOA were invalid. Therefore, the number of target trials within a block was as follows: 36 early-cue-short-SOA, 6 early-cue-medium-SOA, 6 early-cue-longSOA, 36 late-cue-long-SOA, 6 late-cue-medium-SOA, and 6 latecue-short-SOA trials.

In the group without catch trials, a target was displayed on the 32 trials in each block that corresponded to catch trials in the other group. These trials were composed of the same proportions of cue validity and SOA conditions as the remainder of the block. How- ever, they were excluded from the analysis, so that the same number of trials were analyzed in both groups. In other words, we analyzed 96 trials in which a target appeared for both groups.

\section{Design}

The experiment consisted of a 2 (task: detection/discrimination) $\times$ 2 (target uncertainty: without catch trials/with catch trials) $\times 2$ (temporal expectancy: early/late) $\times 3$ (SOA: 400/900/1,400 msec) mixed factor design. Task and target uncertainty were between-subjects variables. Temporal expectancy and SOA were within-subjects variables.

Uncertainty will also be referred to as catch, since uncertainty was induced by the presence of catch trials. Half of the participants completed the detection task, and the remaining half completed the discrimination task. Each task group was divided into two subgroups, referred to as the without catch trial and with catch trial groups. The factorial combination of temporal expectancy and SOA factors defined six conditions that were randomly intermixed among trials within blocks. Reaction times and percentages of errors (misses and mistakes) were the dependent variables. Misses referred to target trials in which no response was made. Mistakes referred to incorrect responses in the discrimination task (e.g., the target was the letter "O" and the key assigned to the target "X" was pressed).

\section{Results}

Trials with correct responses shorter than $100 \mathrm{msec}$ $(0.16 \%)$ or longer than $1,000 \mathrm{msec}(0.84 \%)$, as well as incorrect responses (misses and mistakes), were excluded from the RT analysis. This cutoff was held constant across the experiments. Mean RTs for correct responses and percentages of errors for each experimental condition are shown in Table 1. Only misses are shown for the detection task, since mistakes could not occur in this task.

\section{FIXATION}

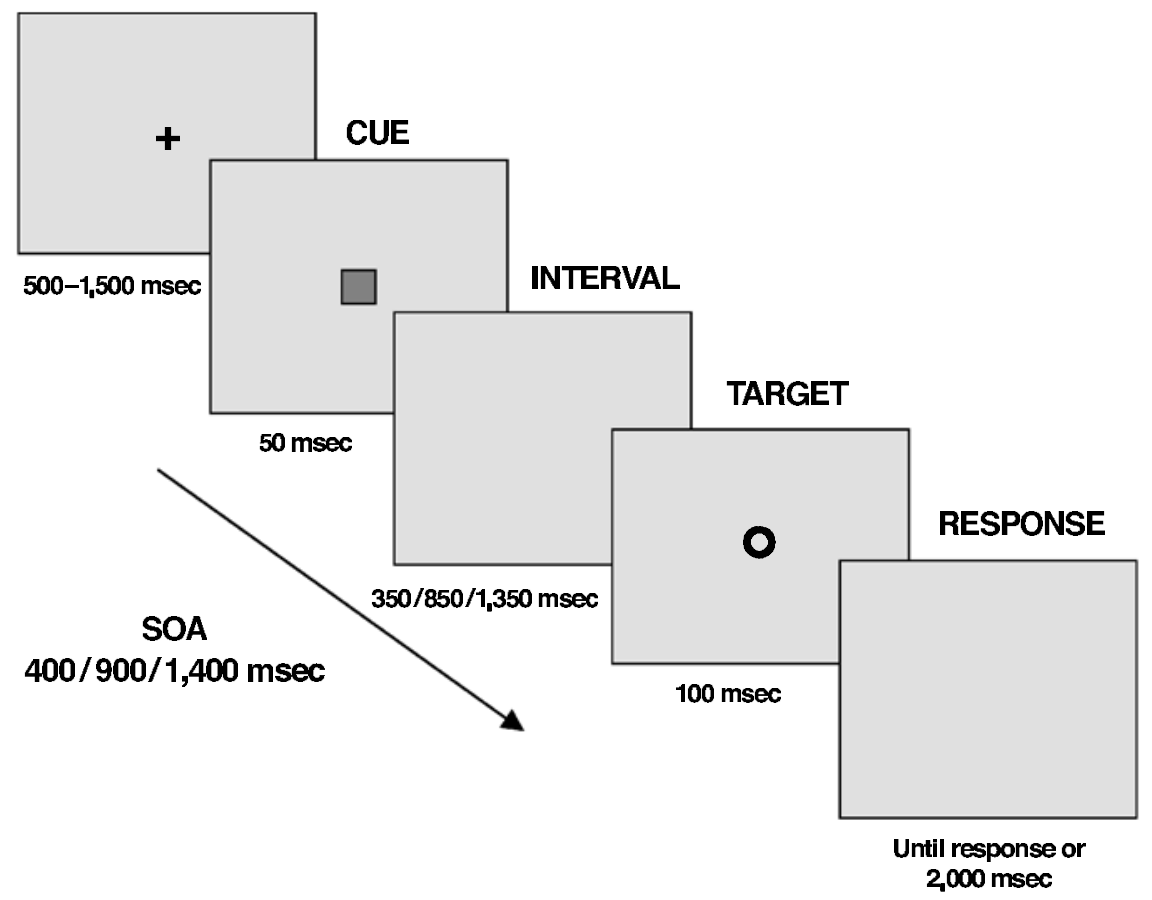

Figure 1. Sequence of events on a trial in Experiment 1. 
Table 1

Mean Correct Response Times (RTs, in Milliseconds) and Percentages of Errors (\% IR, Percentage of Incorrect Responses; \% M, Percentage of Misses) for Each Experimental Condition in the Detection Task and the Discrimination Task, Experiment 1

\begin{tabular}{|c|c|c|c|c|c|c|c|c|c|c|c|c|c|c|c|c|c|c|c|}
\hline \multirow[b]{3}{*}{ Task } & \multirow[b]{3}{*}{ Catch } & \multicolumn{9}{|c|}{ Early Expectancy } & \multicolumn{9}{|c|}{ Late Expectancy } \\
\hline & & \multicolumn{3}{|c|}{$400 \mathrm{msec}$} & \multicolumn{3}{|c|}{$900 \mathrm{msec}$} & \multicolumn{3}{|c|}{$1,400 \mathrm{msec}$} & \multicolumn{3}{|c|}{$400 \mathrm{msec}$} & \multicolumn{3}{|c|}{$900 \mathrm{msec}$} & \multicolumn{3}{|c|}{$1,400 \mathrm{msec}$} \\
\hline & & RT & $\%$ IR & $\% \mathrm{M}$ & RT & $\%$ IR & $\% \mathrm{M}$ & RT & $\%$ IR & $\% \mathrm{M}$ & RT & $\%$ IR & $\% \mathrm{M}$ & RT & $\%$ IR & $\% \mathrm{M}$ & RT & $\%$ IR & $\% \mathrm{M}$ \\
\hline \multirow[t]{2}{*}{ Detection } & With & 358 & & 1.96 & 395 & & 1.03 & 411 & & 2.04 & 376 & & 3.03 & 378 & & 1.54 & 385 & & 1.79 \\
\hline & Without & 345 & & 2.37 & 335 & & 1.03 & 324 & & 1.03 & 371 & & 3.52 & 329 & & 0.00 & 318 & & 1.71 \\
\hline \multirow[t]{2}{*}{ Discrimination } & With & 561 & 1.43 & 0.00 & 569 & 1.46 & 0.00 & 591 & 0.50 & 0.00 & 551 & 1.5 & 0.00 & 565 & 0.50 & 0.00 & 572 & 1.77 & 0.01 \\
\hline & Without & 506 & 1.68 & 0.68 & 498 & 2.79 & 0.00 & 491 & 4.12 & 0.00 & 504 & 4.64 & 0.00 & 488 & 2.42 & 0.51 & 488 & 3.34 & 0.42 \\
\hline
\end{tabular}

Mean RTs for correct responses were submitted to a mixed factor analysis with temporal expectancy (early/ late) and SOA (400/900/1,400 msec) as within-subjects variables and task (detection/discrimination) and uncertainty (without/with catch trials) as between-subjects variables. When the expectancy $\times$ SOA interaction reached significance, temporal-orienting effects were examined more specifically by applying two different analyses of the validity effects. The first analysis focused on how RTs for a given temporal expectancy change across SOA, referred to as expectancy-fixed analyses, or the SOA effect (e.g., Milliken et al., 2003). The second analysis focused on how RTs for a given SOA change across temporal expectancies, referred to as SOA-fixed analyses, or the temporal expectancy effect (e.g., Coull \& Nobre, 1998). The former was accomplished by analyzing the linear and quadratic trends in RTs across SOAs while holding constant the temporal expectancy. The latter consisted of planned comparisons between RTs for valid and invalid trials while holding constant the SOA at either 400 or $1,400 \mathrm{msec}$. Thus, the medium SOA $(900 \mathrm{msec})$ was excluded from the SOA-fixed analyses but was included in linear and quadratic trend analyses across SOAs.
The main effect of task $[F(1,28)=96.11, p<.001]$ confirmed that RTs were shorter in the detection task than in the discrimination task. There was also a significant interaction of task, expectancy, and SOA $[F(2,56)=3.97$, $p<.05]$. To examine this interaction further, analyses were conducted for each task separately.

Detection task. The main effect of catch trials $[F(1,14)=4.38, p<.05]$ revealed faster responses without catch trials than with catch trials.

The interaction between catch trials and expectancy was significant $[F(1,14)=5.39, p<.05]$, so that the slowing of responses with catch trials was more pronounced for the early expectancy than for the late expectancy.

The interaction between catch trials and SOA was also significant $[F(2,28)=18.31, p<.001]$, as is shown in Figure 2. In the group without catch trials, RTs decreased as SOA increased (referred to as a reorienting effect). In the group with catch trials, RTs increased as SOA increased (referred to as a dispreparation effect).

More interesting, the interaction between expectancy and SOA was significant $[F(2,28)=15.51, p<.001]$. This interaction is depicted in Figure 3.

Note that the shortest RTs occurred when temporal expectancy and SOA matched (i.e., early-expectancy-short-

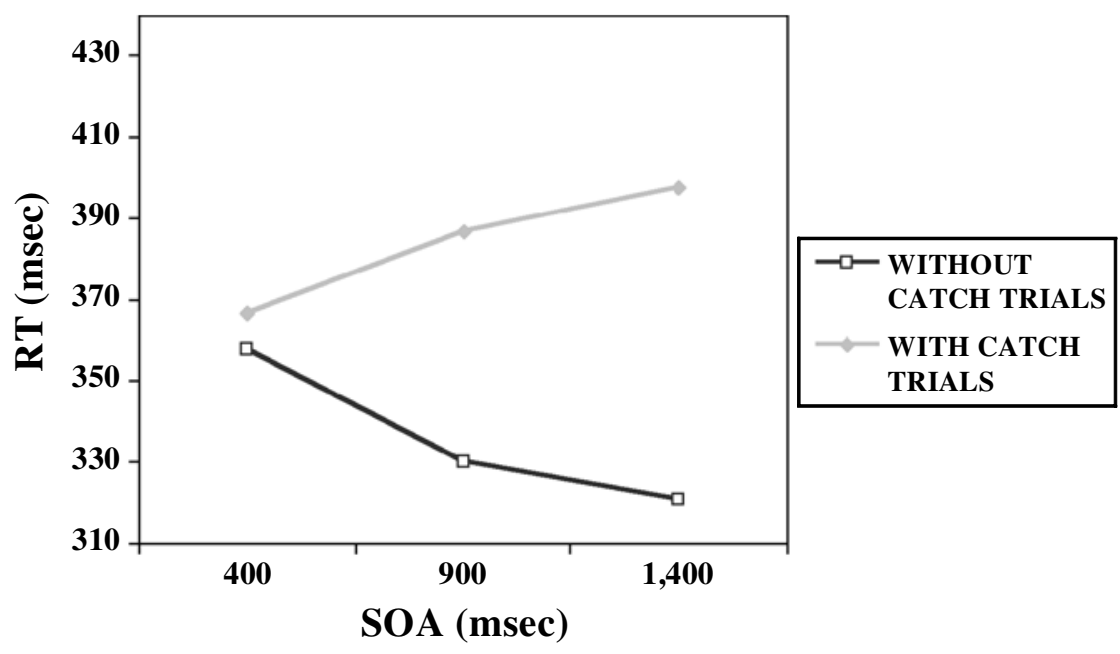

Figure 2. Mean reaction times (RTs) in the detection task of Experiment 1 as a function of catch and stimulus onset asynchrony (SOA). Note the effect of catch trials: reorienting and dispreparation effects for the groups without and with catch trials, respectively. See the text for details. 


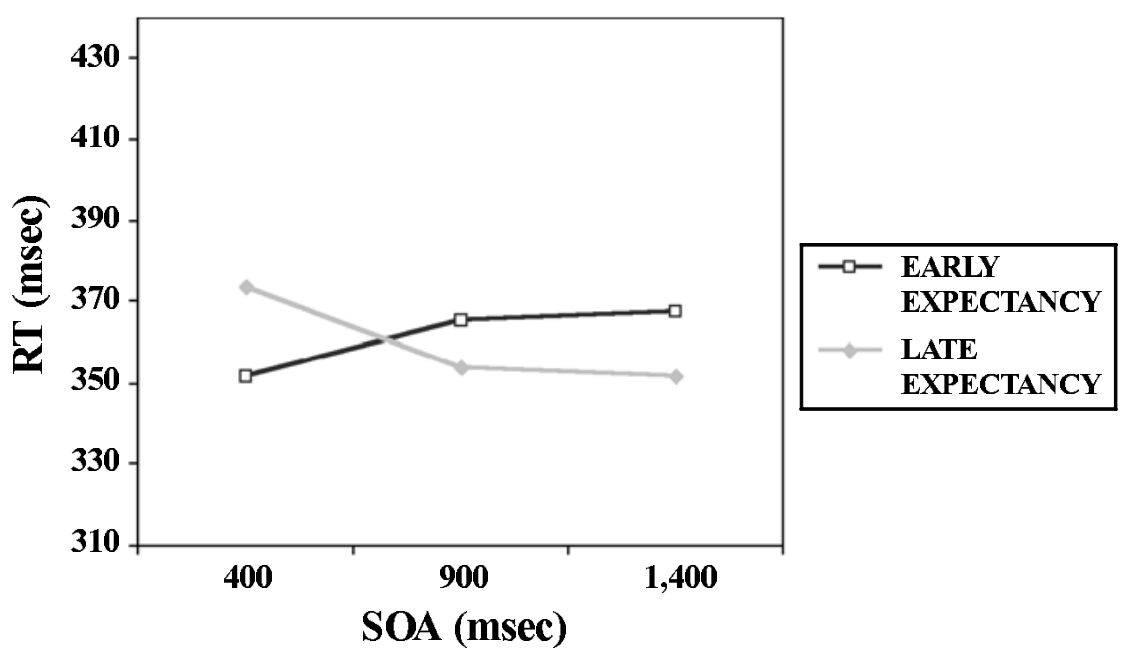

Figure 3. Mean reaction times (RTs) in the detection task of Experiment 1 (with and without catch trials groups collapsed) as a function of temporal expectancy and stimulus onset asynchrony (SOA). Note the validity effects for both early and late expectancies and at short and long SOAs.

SOA and late-expectancy-long-SOA). This observation was confirmed, first, by significant linear trends across SOAs for both early expectancy $[F(1,14)=5.93, p<$ $.05]$ and late expectancy $[F(1,14)=5.93, p<.05]$. The quadratic trend did not reach significance in either case ( $p>.08$ and $p>.2$, respectively). As was predicted (see Table 1), the early-expectancy effect appeared only in the group with catch trials [validity effect, $53 \mathrm{msec} ; F(1,14)=$ $30.11, p<.001]$. On the other hand, the late-expectancy effect was significant only in the group without catch trials [validity effect, $53 \mathrm{msec} ; F(1,14)=17.43, p<.001$ ] . Second, planned comparisons between RTs for valid and invalid trials were significant at the short SOA [validity effect, $22 \mathrm{msec} ; F(1,14)=14.73, p<.01$ ], as well as at the long SOA [validity effect, $16 \mathrm{msec} ; F(1,14)=14.56$, $p<.01$ ]. Importantly (see Table 1 ), catch trials also determined the appearance of validity effects at the long SOA, since they were observed only in the group with catch trials [validity effect, $26 \mathrm{msec} ; F(1,14)=18.79$, $p<.001$, with catch trials; validity effect, $6 \mathrm{msec} ; p>$ .30 , without catch trials].

To summarize, both the expectancy-fixed and the SOA-fixed analyses showed robust temporal-orienting effects, although the effects, as was predicted, were modulated by the presence of catch trials.

Analysis of error percentages (misses) revealed only a main effect of SOA $[F(2,28)=5.22, p<.05]$. Most of the misses occurred at the short SOA $(2.66 \%)$.

Discrimination task. Analyses of the RTs revealed only a significant main effect of catch trials $[F(1,14)=$ $7.2, p<.05]$ and a significant interaction between catch trials and SOA $[F(2,28)=5.69, p<.01]$. The pattern of results was similar to that for the detection task (reorienting and dispreparation effects in the groups without and with catch trials, respectively). Endogenous temporal- orienting effects, revealed by the expectancy $\times$ SOA interaction, were not found in this task.

The analyses of variance (ANOVAs) of mistakes revealed no significant effects. Misses were not analyzed, since they were very rare. No miss rate was higher than $0.7 \%$ (see Table 1 ).

\section{Discussion}

The most important finding in this experiment is that people can direct their attention, voluntarily and flexibly, to specific moments in time. This finding is noteworthy in that it extends the use of attentional-cuing procedures from the domain of space to the domain of time.

The pattern of results that suggests robust voluntary temporal-orienting processes was the co-occurrence of a validity effect for the early- and late-expectancy conditions. The results from Experiment 1 that met this criterion were those in the detection task, and catch trials were shown to be decisive for observing the validity effect for the early expectancy. This criterion is important because it provides additional evidence for voluntary temporal-orienting explanations over alertness explanations. Note that if performance had been faster for validly cued SOAs only in the late-expectancy condition, as has occurred in various prior studies (Coull et al., 2000; Coull \& Nobre, 1998; Coull et al., 2001), it could have been argued that presentation of the cue produced only an alertness effect. Such an alertness effect would be expected to facilitate performance in a rigid manner, with optimal performance occurring at a fixed temporal interval following the cue. However, the studies mentioned reported validity effects at the short SOA, which also points to endogenous effects.

Moreover, we further observed validity effects at both the short and the long SOAs. Again, catch trials were a 
key factor in finding such effects at the long SOA. This result would provide more evidence for the endogenous view, since it shows that temporal orienting is not limited to a fixed temporal interval. Rather, the participants could flexibly focus their attention on different points in time according to their temporal expectancy.

Therefore, Experiment 1 suggests that uncertainty over whether a target will appear on a trial could dictate whether a validity effect occurs for early-expectancy and long-SOA conditions. Thus, the manipulation of uncertainty allowed us to discriminate whether there was a reorienting process that masked validity effects for the early expectancy and the long SOA or there was no temporal orienting at all in those conditions. It appears that reorienting of attention occurred in the group without uncertainty (i.e., without catch trials), whereas an effect we called dispreparation occurred in the group with uncertainty (i.e., with catch trials; see Figure 2). This finding helps to explain why cue validity effects are not always observed, since temporal-orienting processes can be affected by factors other than the match between cue and target.

Specifically, a reorienting process tends to decrease RTs with increasing SOAs. Thus, in the early-expectancy condition, RTs in the long-SOA condition were quite a bit lower than would be expected if RTs depended only on the match between expectancy and SOA. As Coull and Nobre (1998) suggested, participants appear to refocus their attention on the long interval when the target does not appear at the short interval. Indeed, this strategy makes perfect sense when there are no catch trials. Without catch trials, participants can confidently predict the later appearance of the target once the target has not appeared at the short SOA. As such, this reorienting process is likely to eliminate validity effects for the earlyexpectancy and the long-SOA conditions.

In contrast with the reorienting process, dispreparation produces an increase in RTs with increasing SOAs. Dispreparation also makes perfect sense when there are catch trials, in that the conditional probability of target appearance becomes lower with longer SOAs. ${ }^{1}$ Consequently, participants may have relaxed their state of readiness in anticipation of a catch trial. Surprise at the appearance of a target at the long SOA would then augment RTs sharply. Hence, the presence of catch trials prevented the reorienting effect, which in turn allowed us to observe validity effects for the early-expectancy and the long-SOA conditions.

In summary, cue validity is not the only factor that affects temporal orienting. An absence of catch trials appears to encourage a reorienting process that makes validity effects for expect-early cues and long SOAs difficult to observe. In contrast, the presence of catch trials encourages a relaxation of preparedness, which makes cue validity effects difficult to measure for expect-late cues.

Concerning the inclusion of the medium SOA $(900 \mathrm{msec})$, it allowed us to examine the linearity of temporal-orienting effects. In particular, there was an in- crease in RTs as the difference between expected and actual SOAs became larger. Although statistical analyses revealed linear trends instead of quadratic trends in the expectancy functions, such an increment did not seem progressive, since RTs for the 900- and 1,400-msec SOAs were quite similar. This result might suggest that both SOAs could have not been appropriately differentiated, so that, at the time of the medium SOA, the participants achieved (or had already abandoned, in the case of an early cue) the same preparation as that for the long SOA.

On the other hand, temporal-orienting effects were not sufficiently large to be observed in the discrimination task in Experiment 1. Endogenous temporal-orienting effects in a shape discrimination task have been reported in only two prior studies (Griffin et al., 2001; Milliken et al., 2003). This discrepancy could be explained by methodological differences: Milliken et al. manipulated temporal expectancy between subjects, whereas we manipulated temporal expectancy within subjects and within blocks. One possible explanation is that both endogenous temporal orienting (i.e., the voluntary process of attentional preparation in time) and the maintenance in working memory of an arbitrary S-R mapping may interfere with each other. Our shape discrimination task is not demanding in perceptual terms (i.e., the letters " $\mathrm{X}$ " and "O" can be easily discriminated), but in terms of the $\mathrm{S}-\mathrm{R}$ mapping, which is arbitrary. Thus, some processes implied in voluntary temporal orienting could demand controlled processing (e.g., the endogenous generation of the expectancy, or the time estimation processes; Brown, 1985), as well as tasks with complex S-R mappings. Hence, making both demands at the same time could produce interference in central processing.

In brief, our explanation based on interference assumes that temporal orienting, as an endogenous process, demands controlled processing. Moreover, our discrimination task is more demanding than the detection task, in that the $\mathrm{S}-\mathrm{R}$ mapping is more complex (and arbitrary). Hence, the competition for central resources between both demands could result in impaired temporal orienting.

In fact, some of the participants in Experiment 1 mentioned that trying to follow the meaning of the cue distracted them from the discrimination task, so they ignored the temporal information and focused only on the discrimination task. This hypothesis was examined in Experiments 2A and 2B.

\section{EXPERIMENTS 2A AND 2B}

Interference between the demand to accomplish the choice-RT task (arbitrary mapping) and the demand to focus attention in time could explain the absence of temporal-orienting effects for the discrimination task in Experiment 1. Presumably, the resources required for maintaining the $\mathrm{S}-\mathrm{R}$ mapping for the discrimination task prevented simultaneous preparation for expecting a target at a particular temporal interval. 
According to the interference hypothesis, we would expect to observe temporal-orienting effects in choiceRT tasks if the processing demands of preparing for a particular temporal interval were eased. This prediction could be tested by several methods, since there are multiple ways to reduce interference.

For example, temporal expectancy might be manipulated between blocks, rather than within blocks. With this method, participants will not need to generate a new temporal expectancy for each trial, since each experimental block will be composed of homogeneous trials in which expectancy does not change. We tested this possibility in Experiment 2A, in which temporal expectancy was manipulated between blocks for one group of participants and within blocks for a separate group of participants. We expected to observe temporal-orienting effects for the discrimination task in the group for which expectancy was manipulated between blocks, as occurred in Milliken et al. (2003). However, given the results of Experiment 1 , we did not expect to observe temporal-orienting effects for the discrimination task in the group for which expectancy was manipulated within blocks (or at least we expected them to be smaller). In terms of the design of the experiment, then, a significant interaction between temporal expectancy, SOA, and expectancy manipulation (within vs. between blocks) ought to occur.

Following the logic introduced above, another strategy that might be used to find endogenous temporal-orienting effects in a discrimination task would be to facilitate the interpretation of the temporal cue. The cue used in Experiments 1 and 2A linked two different colors to two different time intervals. Since the relation between colors and temporal intervals is arbitrary rather than intuitive, generation of a temporal expectancy with color cues might be relatively resource demanding. By this view, a more easily interpretable cue would require fewer resources and, therefore, might allow for the coexistence of preparation for temporal orienting and maintenance of the $\mathrm{S}-\mathrm{R}$ mapping for the discrimination task. This way, the cue used in Experiment 2B linked two concepts highly related: space and time. We used a line as a cue, the length of which represented the most likely duration of the interval between the cue and the target. A short line indicated that the target was most likely to appear at an early interval, whereas a long line indicated that the target was most likely to appear at a late interval. We would expect to enhance temporal-orienting effects with this more intuitive cue, then, if temporal-orienting effects depend on cue-decoding processes.

We also tested a new measure in Experiment 2B, temporal duration judgments (TDJs), to examine the relation between this measure and temporal-orienting effects. The participants had to estimate the duration of the time interval between the cue and the target. Judgments were retrospective, since the participants were not informed about the estimation task until after they had finished the experiment (see Brown, 1985, for a distinction between retrospective and prospective paradigms of temporal estimation).
This measure could be useful for elucidating whether the participants actually attended to the cue and generated temporal expectancies. We assumed, then, that the participants would have more accurate representations of time intervals if they endogenously generated temporal expectancies. Hence, we expected the accuracy of TDJs to vary directly as a function of temporal-orienting effects in the on-line performance task.

In sum, the aim of Experiments $2 \mathrm{~A}$ and $2 \mathrm{~B}$ was to find temporal-orienting effects in discrimination choice-RT tasks through two different strategies directed to the same goal-that is, to reduce the resources demanded by temporal-orienting processes. In both Experiments $2 \mathrm{~A}$ and $2 \mathrm{~B}$, temporal expectancy was either blocked or mixed within blocks. In Experiment 2B, a more intuitive temporal cue was used. According to the interference hypothesis, we would expect to observe the effect of such manipulations in larger temporal-orienting effects.

\section{Method}

\section{Participants}

Thirty-two students at the University of Granada took part in each experiment for course credit. There were four groups per experiment, each composed of 8 participants. In Experiment $2 \mathrm{~A}$, however, the data from 4 participants (1 participant from each group) were lost for technical reasons.

\section{Apparatus and Stimuli}

The only difference between Experiment $2 \mathrm{~A}$ and Experiment 1 was that the "B" key was not used, since all the participants performed the discrimination task by using the " $Z$ " and "M" keys. The following were the changes introduced in Experiment 2B.

The experiment was run on a computer with a $1,000-\mathrm{MHz}$ Pentium III processor. The cue appeared in the center of the screen and consisted of a red rectangle, $4 \mathrm{~mm}$ in height, subtending a visual angle of $0.38^{\circ}$ at a viewing distance of $60 \mathrm{~cm}$. Its width was either 10 or $22 \mathrm{~mm}\left(0.95^{\circ}\right.$ and $2.1^{\circ}$ of visual angle, respectively).

A time scale printed on a piece of paper was used to collect the TDJs. A $20-\mathrm{cm}$ line was divided into 20 identical parts. The scale ranged from 0 to $2,000 \mathrm{msec}$, in $20 \mathrm{steps}$ of $100 \mathrm{msec}$.

\section{Procedure}

The discrimination task was identical to that in Experiment 1. However, for half of the participants, expectancy was manipulated between blocks. In the between-blocks manipulation, there were one block of 64 practice trials, two blocks of 128 experimental trials with an expect-early cue, and two blocks of 128 experimental trials with an expect-late cue. There was a 1-min interruption for rest at the end of each block. The order of presentation of these four experimental blocks was counterbalanced.

In Experiment 2B, there were two differences from the procedure in Experiment 2A. First, the stimulus used as a temporal cue was either a short or a long red line, indicating an early (for the short line) or a late (for the long line) appearance of the target.

Moreover, at the end of the Experiment 2B, the participants were asked to make an estimate of the duration of both the short $(400 \mathrm{msec})$ and the long $(1,400 \mathrm{msec})$ intervals. The participants were asked to draw two marks, one for each interval, on the scale. Their estimates were constrained to be a multiple of $100 \mathrm{msec}$. In other words, 500 and $600 \mathrm{msec}$, but not $550 \mathrm{msec}$, were eligible estimates.

\section{Design}

Both Experiments 2A and 2B consisted of a 2 (expectancy manipulation: between blocks/within blocks) $\times 2$ (target uncertainty: 
without/with catch trials) $\times 2$ (temporal expectancy: early/late) $\times$ 3 (SOA: 400/900/1,400 msec) mixed factor design. Expectancy manipulation and target uncertainty were between-subjects variables. Temporal expectancy and SOA were within-subjects variables. Moreover, we included type of $\mathrm{cue}^{2}$ (nonintuitive/intuitive) as a between-subjects factor. In Experiment $2 \mathrm{~A}$ the nonintuitive cue (i.e., color as cue) was used, whereas in Experiment 2B the intuitive cue (i.e., length as cue) was used.

In addition to RTs and percentages of errors, in Experiment 2B we collected TDJs about the durations of the short and the long intervals, made by each participant at the end of the experiment.

\section{Results}

Trials with correct responses faster than $100 \mathrm{msec}$ $(0.02 \%$ and $0.01 \%$ for Experiments $2 \mathrm{~A}$ and $2 \mathrm{~B}$, respectively) or slower than $1,000 \mathrm{msec}(1.32 \%$ and $0.74 \%$ for Experiments 2A and 2B, respectively), as well as incorrect responses, were excluded from the RT analysis. Mean RTs for correct responses and percentages of misses and mistakes for each experimental condition in Experiment $2 \mathrm{~A}$ (type of cue: nonintuitive) and in Experiment 2B (type of cue: intuitive) are shown in Table 2.

Mean RTs for correct responses were submitted to a mixed factor analysis with temporal expectancy (early/ late) and SOA (400/900/1,400 msec) as within-subjects variables and type of cue (nonintuitive/intuitive), expectancy manipulation (between blocks/within blocks), and uncertainty (without/with catch trials) as betweensubjects variables.

The type of temporal cue did affect performance overall, as is indicated by the main effect of type of cue $[F(1,52)=$ $4.55, p<.05]$. RTs were shorter when lines with different lengths were used as temporal cues (overall RT in Experiment 2B: $491 \mathrm{msec}$ ) than when squares of different colors were used (overall RT in Experiment 2A: $520 \mathrm{msec}$ ). However, the type of cue did not modulate any interaction between variables, so we did not perform separate analyses for Experiments $2 \mathrm{~A}$ and $2 \mathrm{~B}$. Therefore, the following results include pooled data from Experiments $2 \mathrm{~A}$ and $2 \mathrm{~B}$.

The main effects of catch trials $[F(1,52)=3.90, p<$ $.05]$, temporal expectancy $[F(1,52)=14.54, p<.001]$, and SOA $[F(2,104)=3.85, p<.05]$ were significant, so that RTs for the groups without catch trials were shorter than those for the groups with catch trials, RTs for the early expectancy were shorter than those for the late expectancy, and RTs were shorter for the short than for the long SOA, respectively.

Concerning this expectancy effect, it was modulated by expectancy manipulation [expectancy manipulation $\times$ temporal expectancy; $F(1,52)=16.50, p<.001]$, so that the main effect of temporal expectancy was significant in the between-blocks condition $[F(1,52)=31.06, p<.001]$, but not in the within-blocks condition $(F<1)$. Moreover, the interaction between catch and temporal expectancy $[F(1,52)=7.55, p<.01]$ replicated that for the detection task in Experiment 1, which showed that the slowing produced by the inclusion of catch trials was greater for the early-expectancy than for the late-expectancy condition.

Concerning the SOA variable, the interesting interaction between catch and SOA found in Experiment 1 was replicated $[F(2,104)=25.52, p<.001]$, reflecting the reorienting and dispreparation effects mentioned above. And more interesting, the expectancy $\times$ SOA interaction previously found in the detection task (Experiment 1) was now replicated in the discrimination task $[F(2,104)=50.79, p<.001]$.

However, the expectancy $\times$ SOA interaction depended on expectancy manipulation $[F(2,104)=20.65, p<$ $.001]$. This three-way interaction is depicted in Figure 4.

Note that the typical pattern of RTs observed in temporal orienting experiments (i.e., a crossing between functions for both early and late expectancies) is more evident for the between blocks groups (right panel, with and without catch trials groups collapsed) than for the within-blocks groups (left panel). According to our hypothesis, temporal-orienting effects as revealed by the expectancy $\times$ SOA interaction, although significant for both between- and within-blocks conditions $[F(2,52)=$ $72.88, p<.001$, and $F(2,52)=3.53, p<.05$, respectively], were stronger in the former. Thus, we further analyzed such an interaction for each condition separately.

Further analysis of the between-blocks condition revealed significant linear trends across SOA for both early $[F(1,26)=57.58, p<.001]$ and late $[F(1,26)=$ 26.04, $p<.001]$ expectancy. Also, the quadratic trend

Table 2

Mean Correct Response Times (RTs, in Milliseconds) and Percentages of Errors (\% IR, Percentage of Incorrect Responses; \% M, Percentage of Misses) in Experiment 2A (Type of Cue: Nonintuitive) and Experiment 2B (Type of Cue: Intuitive)

\begin{tabular}{|c|c|c|c|c|c|c|c|c|c|c|c|c|c|c|c|c|c|c|c|c|}
\hline \multirow[b]{3}{*}{ Experiment } & \multirow{3}{*}{$\begin{array}{l}\text { Expectancy } \\
\text { Manipulation }\end{array}$} & \multirow[b]{3}{*}{ Catch } & \multicolumn{9}{|c|}{ Early Expectancy } & \multicolumn{9}{|c|}{ Late Expectancy } \\
\hline & & & \multicolumn{3}{|c|}{$400 \mathrm{msec}$} & \multicolumn{3}{|c|}{$900 \mathrm{msec}$} & \multicolumn{3}{|c|}{$1,400 \mathrm{msec}$} & \multicolumn{3}{|c|}{$400 \mathrm{msec}$} & \multicolumn{3}{|c|}{$900 \mathrm{msec}$} & \multicolumn{3}{|c|}{$1,400 \mathrm{msec}$} \\
\hline & & & RT & $\%$ IR & $\% \mathrm{M}$ & RT & $\%$ IR & $\% \mathrm{M}$ & RT & $\%$ IR & $\% \mathrm{M}$ & RT & $\%$ IR & $\% \mathrm{M}$ & RT & $\% \mathrm{IR}$ & $\% \mathrm{M}$ & RT & $\%$ IR & $\% \mathrm{M}$ \\
\hline \multirow[t]{4}{*}{$2 \mathrm{~A}$} & Within blocks & With & 489 & 3.67 & 0.57 & 505 & 2.29 & 0.58 & 532 & 3.61 & 1.68 & 484 & 3.30 & 0.57 & 521 & 5.30 & 0.56 & 514 & 3.96 & $\overline{1.13}$ \\
\hline & & Without & 501 & 4.04 & 0.91 & 515 & 4.69 & 0.00 & 516 & 5.08 & 0.49 & 513 & 4.12 & 0.50 & 504 & 5.10 & 0.97 & 503 & 3.29 & 0.58 \\
\hline & Between blocks & With & 510 & 3.03 & 0.58 & 557 & 3.51 & 0.49 & 574 & 6.67 & 1.90 & 552 & 3.08 & 0.00 & 545 & 5.71 & 0.00 & 555 & 3.82 & 0.83 \\
\hline & & Without & 481 & 2.86 & 6.28 & 486 & 1.14 & 7.34 & 515 & 1.71 & 6.25 & 574 & 2.46 & 2.26 & 531 & 4.20 & 0.56 & 513 & 3.33 & 0.76 \\
\hline \multirow[t]{4}{*}{$2 \mathrm{~B}$} & Within blocks & With & 503 & 2.19 & 0.49 & 533 & 3.33 & 0.00 & 530 & 5.08 & 0.48 & 517 & 1.36 & 0.00 & 526 & 2.92 & 0.00 & 521 & 2.98 & 0.41 \\
\hline & & Without & 457 & 3.57 & 0.58 & 456 & 3.39 & 0.50 & 457 & 1.00 & 0.51 & 470 & 3.62 & 0.00 & 463 & 4.75 & 0.00 & 451 & 3.85 & 0.2 \\
\hline & Between blocks & With & 454 & 2.66 & 0.17 & 501 & 3.92 & 0.50 & 525 & 1.00 & 0.51 & 518 & 1.46 & 3.94 & 501 & 2.85 & 0.00 & 497 & 2.78 & 0.50 \\
\hline & & Without & 458 & 2.81 & 0.08 & 469 & 3.54 & 0.00 & 474 & 1.96 & 0.00 & 541 & 2.46 & 1.50 & 492 & 2.92 & 0.50 & 472 & 2.98 & 0.08 \\
\hline
\end{tabular}


WITHIN BLOCKS

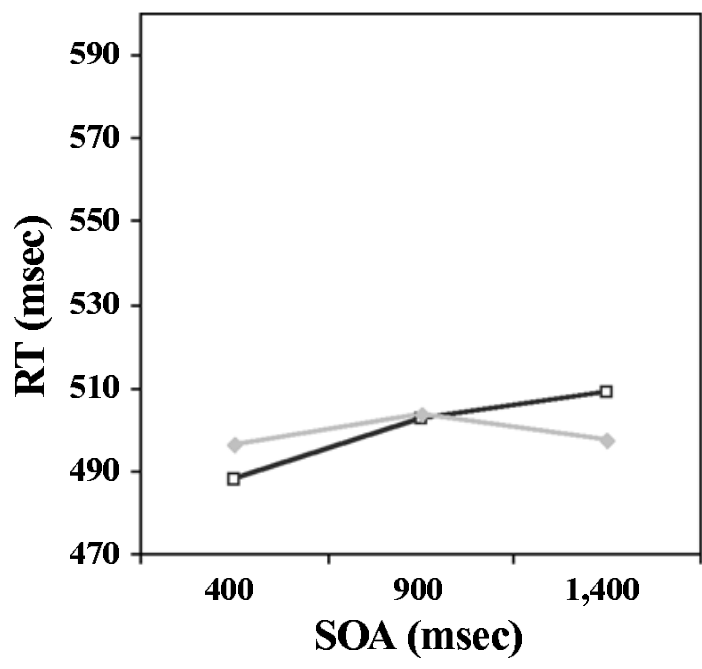

\section{BETWEEN BLOCKS}

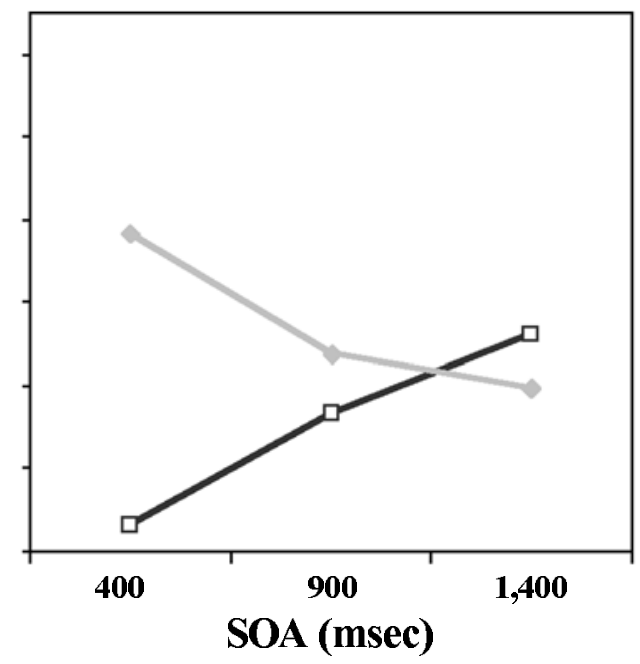

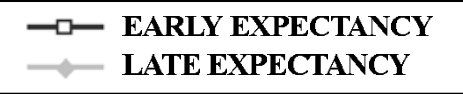

\begin{abstract}
Figure 4. Mean reaction times (RTs) in the discrimination task (Experiments $2 \mathrm{~A}$ and $2 \mathrm{~B}$ collapsed) as a function of temporal expectancy, stimulus onset asynchrony (SOA), and expectancy manipulation, within blocks (left panel) versus between blocks (right panel). Each panel represents data collapsed from two groups: with and without catch trials.
\end{abstract}

was significant for the late expectancy $[F(1,26)=5.26$, $p<.05]$. Furthermore, comparisons between RTs for valid and invalid trials were significant at the short SOA [validity effect: $70 \mathrm{msec} ; F(1,26)=78.18, p<.001$ ] and approached significance at the long SOA [validity effect: $13 \mathrm{msec} ; F(1,26)=3.35, p<.08$ ].

Further analysis of the within-blocks condition revealed a significant linear trend across SOA for the early $[F(1,26)=11.75, p<.01]$, but not for the late $(F<1)$ expectancy. The quadratic trend was not significant in either case ( $p>.2$ and $p>.1$, respectively). Comparisons between RTs for valid and invalid trials were near significance at the short SOA [validity effect: $8 \mathrm{msec}$; $F(1,26)=3.52, p<.07]$ and were significant at the long SOA [validity effect: $12 \mathrm{msec} ; F(1,26)=4.61, p<.05$ ]. As can be seen, these validity effects were quite smaller than those in the between-blocks condition.

Another significant three-way interaction was expectancy manipulation $\times$ catch trials $\times$ expectancy $[F(1,52)=6.16, p<.05]$, so that the catch trials $\times$ expectancy interaction was significant in the betweenblocks condition $[F(1,26)=8.77, p<.01]$, but not in the within-blocks condition $(F<1)$.

The analyses of mistakes (ANOVAs) revealed no significant effects. Misses were not analyzed, since they were very rare.

Analysis of TDJs. This measure was collected only in Experiment 2B, so the following analysis included data only from this experiment. TDJs were submitted to a mixed factor analysis with expectancy manipulation (be- tween blocks/within blocks) and uncertainty (without/with catch trials) as between-subjects variables and SOA (short/long) as a within-subjects variable. The only significant effect was the main effect of SOA $[F(1,28)=$ $112.8, p<.001]$, so that estimates of the 400 -msec interval ( $375 \mathrm{msec}$ on average) were significantly different from estimates of the 1,400-msec interval $(1,103 \mathrm{msec}$ on average).

Our prediction was not supported, since estimates from the within-blocks condition (419 and 1,125 msec for the short and long intervals, respectively) were not significantly less accurate than estimates from the betweenblocks condition (331 and 1,081 msec for the short and long intervals, respectively). Thus, the expectancy manipulation $\times$ SOA interaction was far from significant $(F<1)$.

\section{Discussion}

The most important finding in Experiments $2 \mathrm{~A}$ and $2 \mathrm{~B}$ was the observation of endogenous temporal-orienting effects in a shape discrimination, choice-RT task, as revealed by significant interactions between temporal expectancy and SOA. This finding indicates that temporal orienting is not limited to simple-RT tasks and replicates previous studies that have reported other temporal-orienting effects with choice-RT tasks, such as location discrimination (Kingstone, 1992; Los \& Van den Heuvel, 2001).

Another interesting result concerns the specific experimental conditions in which temporal-orienting effects are more clearly observed in discrimination tasks. 
The results of both experiments suggest that the way temporal expectancy is manipulated can be critical. In particular, temporal-orienting effects were hardly found when expectancy was manipulated trial by trial, a result in line with the findings from the discrimination task in Experiment 1. However, as was predicted, temporalorienting effects were remarkably enhanced when expectancy was manipulated between blocks. We suggest that generating an expectancy that a target will occur within a specific temporal window while maintaining an arbitrary $\mathrm{S}-\mathrm{R}$ mapping for our discrimination task is highly demanding to the attentional system. Consequently, we found smaller temporal-orienting effects when temporal expectancy cues were manipulated on a trial-bytrial base than when temporal expectancy was constant within a block of trials, presumably because fewer resources were needed to generate the expectancy consistently across a block.

Concerning the manipulation based on the type of the cue, overall RT in Experiment 2B was shorter than that in Experiment 2A. This result suggests that processes of symbolic decoding of the more intuitive cue demanded fewer resources in Experiment 2B than in Experiment 2A, thus freeing resources for other aspects of task performance. However, type of cue did not interact with any factor, suggesting that this manipulation was not decisive in observing temporal orienting in discrimination tasks. Reducing the resources required by using a more intuitive cue, then, did not lead to larger temporal-orienting effects. However, other methods could facilitate temporal orienting more successfully. For example, participants might be allowed more time to generate a preparatory set for a given time interval. Given that the cue was displayed for $50 \mathrm{msec}$ in these experiments and the shortest SOA was $400 \mathrm{msec}$, it remains possible that temporal-orienting effects were obscured in the discrimination task because the participants had insufficient time to generate a temporal expectancy (see Kingstone, 1992, or Los \& Van den Heuvel, 2001, for longer exposure times of the temporal cue). This is an issue that ought to be addressed in future research.

On the other hand, TDJs did not prove to be a reliable index of temporal-orienting effects, given that this variable did not discriminate between the conditions that did (between-blocks groups) and did not (within-blocks groups) produce remarkable temporal-orienting effects. Contrary to our hypothesis, estimations were not more accurate in the condition that showed stronger temporalorienting effects. However, this measurement probably was not reliable enough, because we registered just one estimate from each participant. Other studies that have measured TDJs (e.g., Chen \& O'Neill, 2001) have registered one estimate after each trial, so that the number of estimates per participant was considerably higher than that in our study.

Isolating endogenous from nonendogenous influences on temporal orienting: An analysis of sequential effects. So far, we have shown that temporal orienting of attention can be observed in both a detection
simple-RT task and a discrimination choice-RT task, for both early and late expectancies, and at short and long SOAs. These data point to the endogenous nature of our temporal-orienting effects, in line with the results observed by Nobre and co-workers (e.g., Coull et al., 2000; Coull \& Nobre, 1998; Miniussi et al., 1999). However, other researchers have studied similar temporal-orienting effects, which are, according to them, nonendogenous (Los \& Van den Heuvel, 2001).

Thus, it seems appropriate to disentangle endogenous from nonendogenousinfluences on our temporal-orienting effects, in order to test thoroughly our main hypothesis that such effects are produced by an endogenous mechanism. Moreover, this analysis will allow us to link studies on temporal orienting with the classical literature on FP and preparation.

Studies of preparation that have manipulated FP duration usually find asymmetrical sequential effects (Niemi \& Näätänen, 1981). FP is defined as the time interval between a warning signal and a target. Therefore, if we consider that FP and SOA are practically the same variable, it makes sense to expect that our SOA manipulation additionally produced such effects. Asymmetrical sequential effects imply that RTs are lengthened when the previous FP was longer than the current FP. In other words, RTs at a short current SOA are longer when the previous SOA was long rather than short. Typically, this relation is not symmetrical, because RTs at a long current SOA are not different when the previous SOA was either short or long.

Hence, our data could be contaminated by such an effect, so that the expectancy $\times$ SOA interaction might be due to asymmetrical sequential effects, rather than to temporal cuing. ${ }^{3}$ Thus, to distinguish between these two causes, it is important to argue that there exists an endogenous mechanism of attention underlying our temporalorienting effects, since Los and Van den Heuvel (2001) have presented evidence that sequential effects are produced by unintentional processes. Therefore, we will consider sequential effects as nonendogenous influences, to put them in opposition to our account in which temporalorienting effects are based on endogenous influences (but see Alegria, 1975, or Niemi \& Näätänen, 1981, for endogenous accounts of sequential effects).

In order to control for potential sequential effects, we made a reanalysis of the data of the experiments reported that included the SOA of the previous trial as a factornamely, $\mathrm{SOA}_{n-1}$ (the current SOA will be referred to as SOA). Thus, the contribution of automatic sequential effects on temporal orienting could be estimated.

The design was then modified to add the $\mathrm{SOA}_{n-1}$ factor. Given that catch trials can also contribute to sequential effects, the effect of a catch trial on a subsequent trial was considered in this factor (see note 3 ). However, data for the medium SOA and the medium $\mathrm{SOA}_{n-1}$ were excluded from the analyses, due to insufficient observations. Moreover, only the groups with catch trials were analyzed, for two main reasons. First, the sequential effects of catch trials could not be analyzed in the groups 
without catch trials. On the other hand, as is shown by our results, validity effects for both SOAs are better observed in the groups with catch trials.

As was stated above, asymmetrical sequential effects would imply an interaction between $\mathrm{SOA}_{n-1}$ and SOA, so that RTs at the short SOA would be higher for the long than for the short $\mathrm{SOA}_{n-1}$, whereas RTs at the long SOA would not be very different for the long and the short $\mathrm{SOA}_{n-1}$. As was said before, the interaction between temporal expectancy and SOA will be the hallmark of temporal-orienting effects.

Again, analyses were conducted for each task separately. For the detection task, RTs for correct responses were submitted to a repeated measures analysis with $\mathrm{SOA}_{n-1}$ (short/long/catch trial), temporal expectancy (early/late), and SOA (400/1,400 msec) as within-subjects variables. For the discrimination task, the analysis also included expectancy manipulation (between blocks/within blocks) as a between-subjects variable.

Detection task. The detection task analysis included one group with catch trials in which temporal expectancy was manipulated within blocks (Experiment 1).

A significant interaction between $\mathrm{SOA}_{n-1}$ and SOA $[F(2,14)=6.27, p<.01]$ showed the asymmetrical sequential effects mentioned. As was expected, the interaction between expectancy and SOA was also significant $[F(1,7)=20.22, p<.01]$. Crucially, the interaction between $\mathrm{SOA}_{n-1}$, expectancy, and SOA was not significant $[F(2,14)=1.27, p>.3]$.

As can be seen in Figure 5, the validity effects related to intentional temporal expectancies hold separately for the short, the long, and the catch trial $\mathrm{SOA}_{n-1}$ conditions. Therefore, although sequential and temporal-orienting effects affected RT, both contributions appear to be independent of each other. This result suggests that endogenous temporal orienting cannot be reduced to explanations based on sequential effects.

Discrimination task. The discrimination task analysis included three within-blocks groups (Experiments 1,2A, and 2B) and two between-blocks groups (Experiments 2A and $2 \mathrm{~B}$ ). All the groups had catch trials.

A significant interaction between $\mathrm{SOA}_{n-1}$ and SOA $[F(2,70)=13.65, p<.001]$ replicated the sequential effects obtained in the detection task. Moreover, temporalorienting effects were denoted by the interaction between expectancy and SOA $[F(1,35)=21.23, p<.001]$. Importantly, the three-way interaction between $\mathrm{SOA}_{n-1}$, expectancy, and SOA was again not significant $[F(2,70)=$ $1.86, p>.16$ ], as can be observed in Figure 6 .

Thus, the findings of the discrimination task replicated those of the detection task, in which temporal-orienting effects were observed independently of the effects produced by the SOA in the previous trial.

Interestingly, the data from the discrimination groups allowed us to explore the effect of blocking. The marginally significant interactions between expectancy manipulation, $\mathrm{SOA}_{n-1}$, and $\mathrm{SOA}[F(2,70)=2.79, p<.07]$ and expectancy manipulation, temporal expectancy, and SOA $[F(1,35)=4.03, p<.05]$ revealed that the size of both sequential and temporal-orienting effects depended on expectancy manipulation. In particular, the size of both effects was larger in the between-blocks than in the within-blocks groups.

In conclusion, analyses from detection and discrimination tasks disentangled temporal-orienting effects related to endogenous processes from other effects more

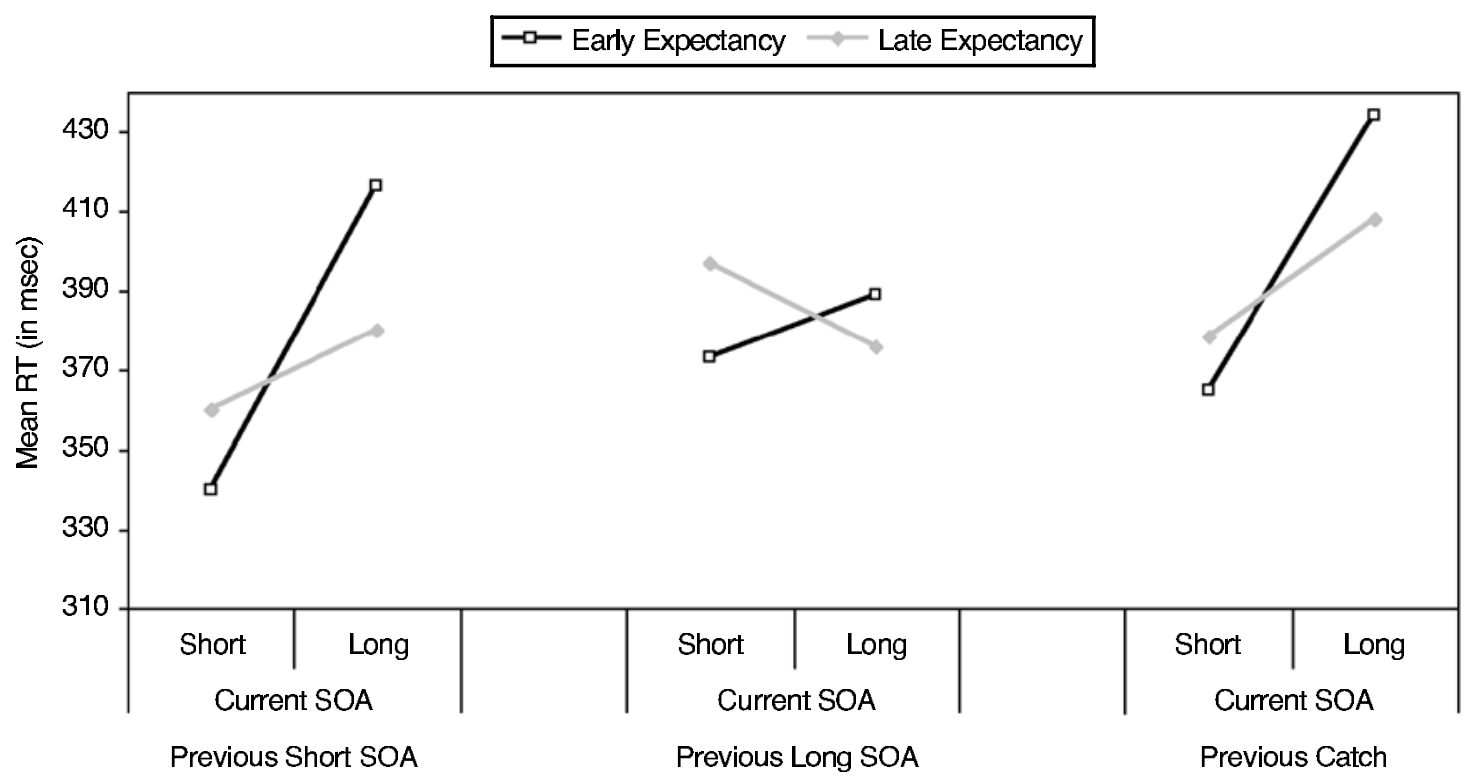

Figure 5. Mean reaction times (RTs) in the detection task as a function of temporal expectancy, stimulus onset asynchrony (SOA), and the SOA in the previous trial. Note that the temporal-orienting effects were independent of the sequential effects produced by the previous trial SOA. 


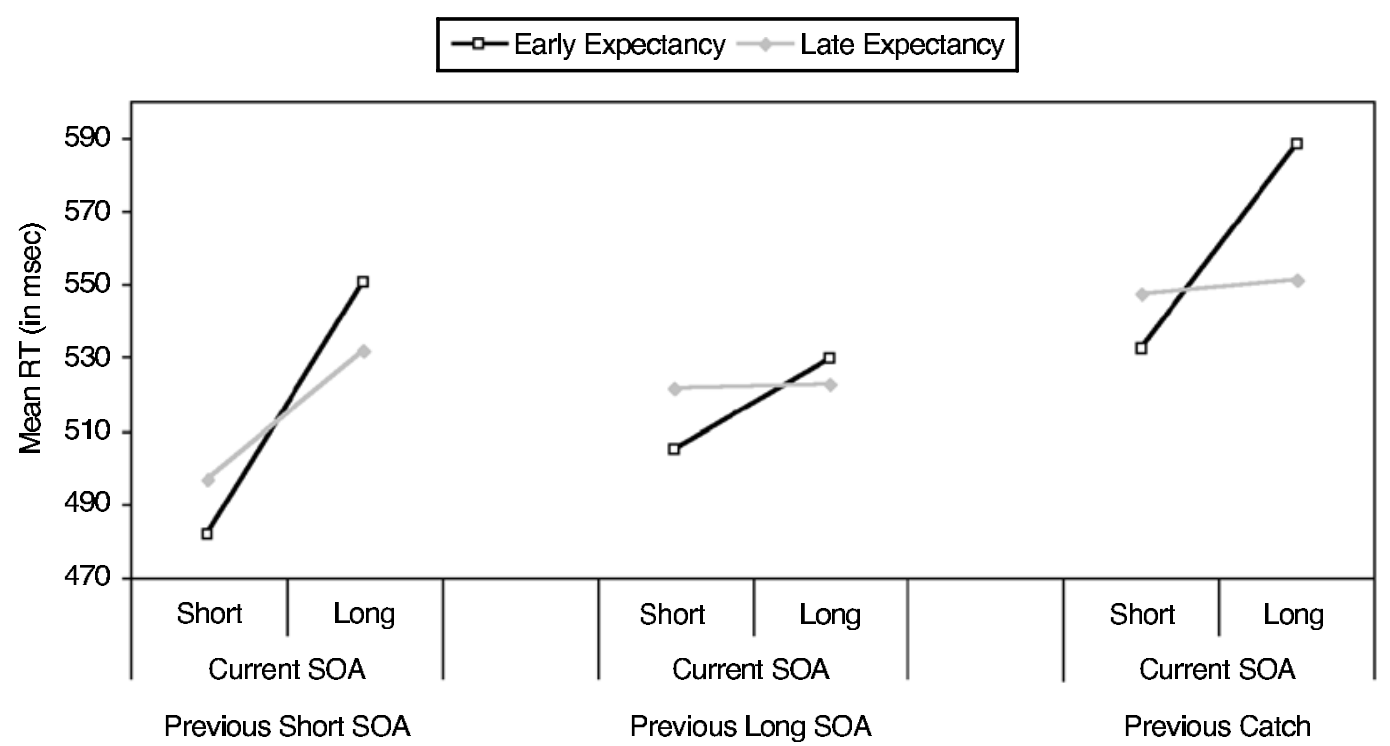

\begin{abstract}
Figure 6. Mean reaction times (RTs) in the discrimination task as a function of temporal expectancy, stimulus onset asynchrony (SOA), and the SOA in the previous trial. Note that the temporal-orienting effects were independent of the sequential effects produced by the previous trial SOA.
\end{abstract}

closely related to automatic processes, such as sequential effects. These results provide converging evidence suggesting that temporal-orienting effects cannot be adequately explained by nonendogenous accounts based on sequential effects. Therefore, it seems also necessary to consider an endogenous mechanism of attention, which is guided by the participant's expectancies.

\section{GENERAL DISCUSSION}

The results of the three experiments suggest that uncertainty over whether a target will appear is an important factor in determining whether temporal-orienting effects occur for an early-expectancy condition. When catch trials are present, they appear to prevent the reorienting of attention to longer intervals on invalid trials. Thus, for the groups without catch trials, the reorienting of attention seemed to eliminate validity effects for the early-expectancy condition and at the long SOA. In this sense, such groups replicated the results from studies in which no temporal-orienting effects were obtained for an early-expectancy condition (Coull et al., 2000; Coull \& Nobre, 1998; Coull et al., 2001). For the groups with catch trials, however, uncertainty induced a dispreparation effect, which allowed validity effects to be measured for the early-expectancy and long-SOA conditions. Thereby, the groups with uncertainty replicated the results from studies that reported temporal-orienting effects for an early expectancy (Milliken et al., 2003; Miniussi et al., 1999).

Together, the results indicate that uncertainty generated by catch trials modulates temporal-orienting effects. Hence, an interesting question concerns exactly how uncertainty over the presence of a target affects processing.
We proposed that a dispreparation process occurs when there are catch trials in the experiment and that it prevents a reorienting process, thus allowing validity effects to be measured for both the early expectancy and the long SOA.

With this framework in mind, it is worth considering the data from the late-expectancy condition (see Table 1: detection task, catch trials group of Experiment 1). For the late expectancy, one would expect shorter RTs at the long SOA $(1,400 \mathrm{msec})$ than at the short SOA $(400 \mathrm{msec})$ if, in fact, endogenous temporal-orienting processes underlie performance. However, note that the RTs were very similar across SOAs $(376,378$, and $385 \mathrm{msec}$ for the short, medium, and long SOAs, respectively). This pattern was also observed in other groups with catch trials from Experiments $2 \mathrm{~A}$ and $2 \mathrm{~B}$, as can be seen in Table 2 (between-blocks and within-blocks groups, respectively). How might this type of results be explained?

We propose that this result was the consequence of two antagonist processes-namely, an orienting process produced by a late temporal cue, which decreased RTs for the long SOA, and a dispreparation process produced by catch trials, which increased RTs. Together, these two processes would produce no effect of SOA in the lateexpectancy condition. Subsequent experiments that manipulate both temporal expectancy and uncertainty in a graded fashion (e.g., by varying the proportion of catch trials) might provide converging support for this proposal.

On the other hand, no significant temporal-orienting effects were observed in the discrimination task in Experiment 1 . We proposed that this result occurred because endogenous temporal orienting competes for central resources, which are also needed to accomplish the arbitrary $\mathrm{S}-\mathrm{R}$ mapping used in the discrimination task. 
Accordingly, in Experiments $2 \mathrm{~A}$ and $2 \mathrm{~B}$, we eased the resource demands associated with the generation of temporal expectancy by manipulating it between blocks, rather than in a trial-to-trial within-blocks manner. Indeed, a clearer temporal-orienting effect was observed when temporal expectancy was manipulated between blocks. We conclude that endogenous temporal orienting in discrimination tasks can occur reliably, but the magnitude of such effects depends critically on how temporal expectancy is manipulated.

Therefore, another noteworthy result was the finding of a factor that clearly determined the size of the temporalorienting effects in our discrimination task. Experiments $2 \mathrm{~A}$ and $2 \mathrm{~B}$ showed that such effects are maximized when temporal expectancy is blocked. We have suggested that the dependency of temporal-orienting effects on expectancy blocking might be due to the interference between expectancy generation (on each trial) and the maintenance in working memory of the discrimination task set (arbitrary S-R mapping). Hence, if expectancy is manipulated within blocks, with the easy $\mathrm{S}-\mathrm{R}$ mapping for the detection task becoming more complex in the discrimination task, temporal-orienting effects were not observed (discrimination task of Experiment 1) or, at least, were more difficult to observe (Experiments 2A and $2 \mathrm{~B}$ collapsed). Thus, the use of discrimination tasks provided important information regarding the nature of the processes underlying temporal orienting. The fact that such processes could be impaired with increments in other simultaneous demands indicates their controlled nature. It appears that endogenously focusing attention on time is a demanding task in a within-blocks context.

Moreover, the finding of temporal-orienting effects in a shape discrimination task raises the theoretical question of whether temporal attention affects early or late stages of processing (i.e., modulation of perceptual or motor processing, respectively). Previous research with detection tasks has suggested that temporal orienting implies mainly preparation of motor processes (Coull et al., 2000; Coull \& Nobre, 1998; Miniussi et al., 1999), whereas the present findings with discrimination tasks might open the possibility that temporal attention also enhances perceptual processes. However, the motor account cannot be ruled out exclusively on the basis on our RT data.

Hence, the use of shape discrimination tasks could be useful for tracking the physiological modulations of perceptual processing produced by temporal attention. It remains possible that cerebral activation patterns normally found in temporal-orienting studies (Coull et al., 2000; Coull \& Nobre, 1998; Miniussi et al., 1999) are partially due to the specific demands of the task at hand (i.e., detection). Thus, motor areas may be involved in detection tasks, whereas perceptual areas may be more involved in discrimination than in detection tasks.

As a final point, the analyses of sequential effects have revealed the contribution of unintentional factors to temporal orienting or preparation. However, these analyses have also revealed that temporal orienting can occur independently of sequential unintentional effects. There- fore, the temporal-orienting effects reported here cannot be reduced to a nonendogenous preparation. In conclusion, it is likely that both endogenous and automatic factors contribute to temporal orienting and preparation. The primacy of one in particular might well depend on specific demands in the context of the experiment.

\section{REFERENCES}

Alegria, J. (1975). Sequential effects of foreperiod duration: Some strategical factors in tasks involving time uncertainty. In P. M. A. Rabbitt \& S. Dornic (Eds.), Attention and performance $V$ (pp. 1-11). London: Academic Press.

BERTELSON, P. (1967). The time course of preparation. Quarterly Journal of Experimental Psychology, 19, 272-279.

Brown, S.W. (1985). Time perception and attention: The effects of prospective versus retrospective paradigms and task demands on perceived duration. Perception \& Psychophysics, 38, 115-124.

Chen, C., \& O'Neill, P. (2001). Processing demand modulates the effects of spatial attention on the judged duration of a brief stimulus. Perception \& Psychophysics, 63, 1229-1238.

Coull, J. T., Frith, C. D., BüChel, C., \& Nobre, A. C. (2000). Orienting attention in time: Behavioural and neuroanatomical distinction between exogenous and endogenous shifts. Neuropsychologia, 38, 808-819.

COUlL, J. T., \& NoBre, A. C. (1998). Where and when to pay attention: The neural systems for directing attention to spatial locations and to time intervals as revealed by both PET and PMR. Journal of Neuroscience, 18, 7426-7435.

Coull, J. T., Nobre, A. C., \& Frith, C. D. (2001). The noradrenergic alpha2 agonist clonidine modulates behavioural and neuroanatomical correlates of human attentional orienting and alerting. Cerebral Cortex, 11, 73-84.

GARNER, W. R. (1987). Location and color as cuing dimensions in contingent classification. Perception \& Psychophysics, 41, 202-210.

Griffin, I. C., Miniussi, C., \& Nobre, A. C. (2001). Orienting attention in time. Frontiers in Bioscience, 6, 660-671.

KARLIN, L. (1959). Reaction time as a function of foreperiod duration and variability. Journal of Experimental Psychology, 58, 185-191.

Kingstone, A. (1992). Combining expectancies. Quarterly Journal of Experimental Psychology, 44A, 69-104.

KuBOVY, M. (1981). Concurrent-pitch segregation and the theory of indispensable attributes. In M. Kubovy \& J. R. Pomerantz (Eds.), Perceptual organization (pp. 55-98). Hillsdale, NJ: Erlbaum.

LABERGE, D. (1995). Attentional processing: The brain's art of mindfulness. Cambridge, MA: Harvard University Press.

LOS, S. A., \& VAN DEN HEUVEL, C. E. (2001). Intentional and unintentional contributions to nonspecific preparation during reaction time foreperiods. Journal of Experimental Psychology: Human Perception \& Performance, 27, 370-386.

Loveless, N. E., \& SANFORD, A. J. (1974). Effects of age on the contingent negative variation and preparatory set in a reaction-time task. Journal of Gerontology, 29, 52-63.

MCAulEy, J. D., \& KiDD, G. R. (1998). Effect of deviations from temporal expectations on tempo discrimination of isochronous tone sequences. Journal of Experimental Psychology: Human Perception \& Performance, 24, 1786-1800.

Milliken, B., \& LuPiáñEZ, J. (2000, March). Strategic influences on inhibition of return: The effect of SOA proportion manipulations in discrimination and detection tasks. Paper presented at the III Congreso de la Sociedad Española de Psicología Experimental, Barcelona.

Milliken, B., Lupiáñ̃e Z, J., Roberts, M., \& STEvanovski, B. (2003). Orienting in space and time: Joint contributions to exogenous spatial cuing effects. Psychonomic Bulletin \& Review, 10, 877-883.

Miniussi, C., Wilding, E. L., Coull, J. T., \& Nobre, A. C. (1999). Orienting attention in time: Modulation of brain potentials. Brain, 122, 1507-1518.

NIEMI, P., \& NÄÄTÄNEN, R. (1981). Foreperiod and simple reaction time. Psychological Bulletin, 89, 133-162.

Posner, M. I. (1978). Chronometric explorations of mind. Hillsdale, NJ: Erlbaum.

Posner, M.I., Nissen, M.J., \& Ogden, W.C. (1978). Attended and un- 
attended processing modes: The role of set for spatial location. In H. L. Pick \& I. J. Saltzman (Eds.), Models of perceiving and information processing (pp. 137-157). Hillsdale, NJ: Erlbaum.

Posner, M. I., \& RAICHLE, M. E. (1994). Images of mind. New York: Scientific American Library.

Posner, M. I., SNyder, C. R. R., \& DAvidson, B. J. (1980). Attention and the detection of signals. Journal of Experimental Psychology: General, 109, 160-174.

SCHNEIDER, W. (1988). Micro Experimental Laboratory: An integrated system for IBM PC compatibles. Behavior Research Methods, Instruments, \& Computers, 20, 206-271.

Shulman, G. L., SHEehy, J. B., \& Wilson, J. (1986). Gradients of spatial attention. Acta Psychologica, 61, 167-181.

Woodrow, H. (1914). The measurement of attention. Psychological Monographs, 17, 1-158.

\section{NOTES}

1. In the groups with catch trials, the conditional probabilities that an early-cued target will occur at the short, medium, and long SOAs are $.56, .21$, and .27 , respectively.

2. Although the two experiments were run independently, the data are presented as a between-subjects variable for the sake of simplicity. As can be seen, the data for the two experiments were quite similar.

3. We thank Sander A. Los for his suggestions on this point.

(Manuscript received November 29, 2002; revision accepted for publication July 14, 2003.) 